\title{
Coming Clean: Understanding and Mitigating Optical Contamination and Laser Induced Damage in Advanced LIGO
}

\author{
Kaitlan E. Gushwa and Calum I. Torrie \\ aLIGO Laboratory, California Institute of Technology, 1200 E. California Blvd., \\ Pasadena, CA USA 91125
}

\begin{abstract}
The cleanliness of optical surfaces is of great concern as the Advanced Laser Interferometer Gravitational-Wave Observatory (LIGO) project transitions from installation to operation at full power. More particulates than expected were observed on and near the core optics as a result of assembly and installation work, prompting a re-evaluation of longheld contamination control practices. Even low particulate levels can potentially damage the fused silica optics and reduce overall interferometer sensitivity. These risks are mitigated from a combination of the following approaches: quantifying the extent of the contamination, identifying its sources, improving practices to reduce the generation of particulates, introducing a non-contact in-situ cleaning technique for suspended optics in air, qualifying cleanliness levels against induced damage, and developing methods for remotely measuring and cleaning suspended optics under vacuum. While significant progress has been made in understanding and mitigating contamination, and thus, protecting the optics from losses and damage, there is still more work to be done to reach ultimate performance requirements.
\end{abstract}

Keywords: LIGO, gravitational waves, ultra-high vacuum system, coated fused silica optics, damage, absorption and scatter, contamination control, particulate cleanliness level, obscuration ratio.

\section{INTRODUCTION}

In his 1916 General Theory of Relativity [1], Einstein predicted the existence of gravitational waves (GW). If space-time is thought of as a fabric, then gravity is the curvature or warpage of the fabric around large amounts of mass or energy. Accelerating masses generate perturbations in the curvature that propagate outward at the speed of light. These ripples in the fabric of space-time are called gravitational waves. There are a number of predicted sources of gravitational waves, such as supernova explosions (the death of giant stars), pulsars (pulsating neutron stars), and from the mergers of black holes or neutron stars [2].

There is strong indirect evidence for the existence of gravitational waves. The 1993 Nobel Prize in physics was awarded for measurements of the Hulse-Taylor binary pulsar, and the verification of an orbital decay rate consistent with that expected from gravitational wave emission [3], [4]. Today, collaborations around the world are working to directly detect gravitational waves for the first time. Achieving this goal will both test relativistic gravitation in the strong-field limit, and allow for the observation of much of the universe that is now obscured. To date, astronomy has relied on electromagnetic waves to study the cosmos. Unlike electromagnetic waves, gravitational waves travel unimpeded through matter and space, carrying information about astrophysical objects and events that could not be detected by other means. The direct detection of gravitational waves offers an entirely new view of the universe. Detecting a gravitational wave is like looking for an atom-size change in distance between the ends of a ruler $\sim$ ten times as long as the distance between the Earth and the Moon [5].

One technique for detecting gravitational waves is laser interferometry. In a Michelson interferometer, see Figure 1, a laser incident on a beamsplitter (BS) is evenly divided between two arms. Mirrors at the end of the arms (ETM) reflect the light back to the beamsplitter, and the combined interference pattern is detected at the photodiode (PD). A passing gravitational wave stretches and squeezes space-time, changing the relative lengths of the arm cavities, thus changing the interference pattern at the photodetector (PD). Figure 1 indicates the full optical configuration for the Advanced LIGO interferometer. The basis of the design is a modified Michelson interferometer, with a Fabry-Perot resonant cavity in each arm to build up the phase shift produced by an arm length change. Power recycling is another standard feature of such interferometers: the power recycling mirror (PRM) forms a resonant cavity between the laser source and the

Laser-Induced Damage in Optical Materials: 2014, edited by Gregory J. Exarhos, Vitaly E. Gruzdev, Joseph A. Menapace, Detlev Ristau, MJ Soileau, Proc. of SPIE Vol. 9237, 923702 • (C 2014

SPIE - CCC code: $0277-786$ X/14/\$18 - doi: 10.1117/12.2066909 
Michelson to increase the effective laser power [6]. LIGO consists of two ground-based 4km Michelson interferometers; one in Livingston Parish, Louisiana and one on the Hanford site in Washington State. The Initial LIGO detectors were completed in 2000. The design sensitivity was achieved in 2005, and the detectors ran for several years [7]. Despite no confirmed detection, Initial LIGO has produced astrophysically interesting results see e.g. [8], [9], [10], [11]. The LIGO project was planned from the beginning to realize several generations of gravitational wave detectors, with ever improving sensitivity. Installation began in 2010 on the Advanced LIGO detectors, the second generation of interferometers, and after $\sim 4$ years, is now complete. The first observing run with both interferometers working at the same sensitivity level is currently planned for the summer of 2015. Once fully operational, Advanced LIGO is expected to be 10x more sensitive and will have 1000x greater observable volume than the Initial LIGO detectors.

The optics in the LIGO detectors are suspended as pendulums under vacuum to isolate from noise sources such as ground vibrations and air pressure fluctuations. The ultra-high vacuum (UHV) system operates at pressures down to 1x10-9 Torr. LIGO's fused silica optics are $34 \mathrm{~cm}$ diameter x $20 \mathrm{~cm}$ thick, weigh $40 \mathrm{~kg}$, and have a High Reflectance (HR) coating consisting of alternating layers of silica \& titanium doped tantala. The optics are designed to have very low intrinsic absorption and scatter; however, they are also highly sensitive to scatter and absorption loss induced by particulate contamination.

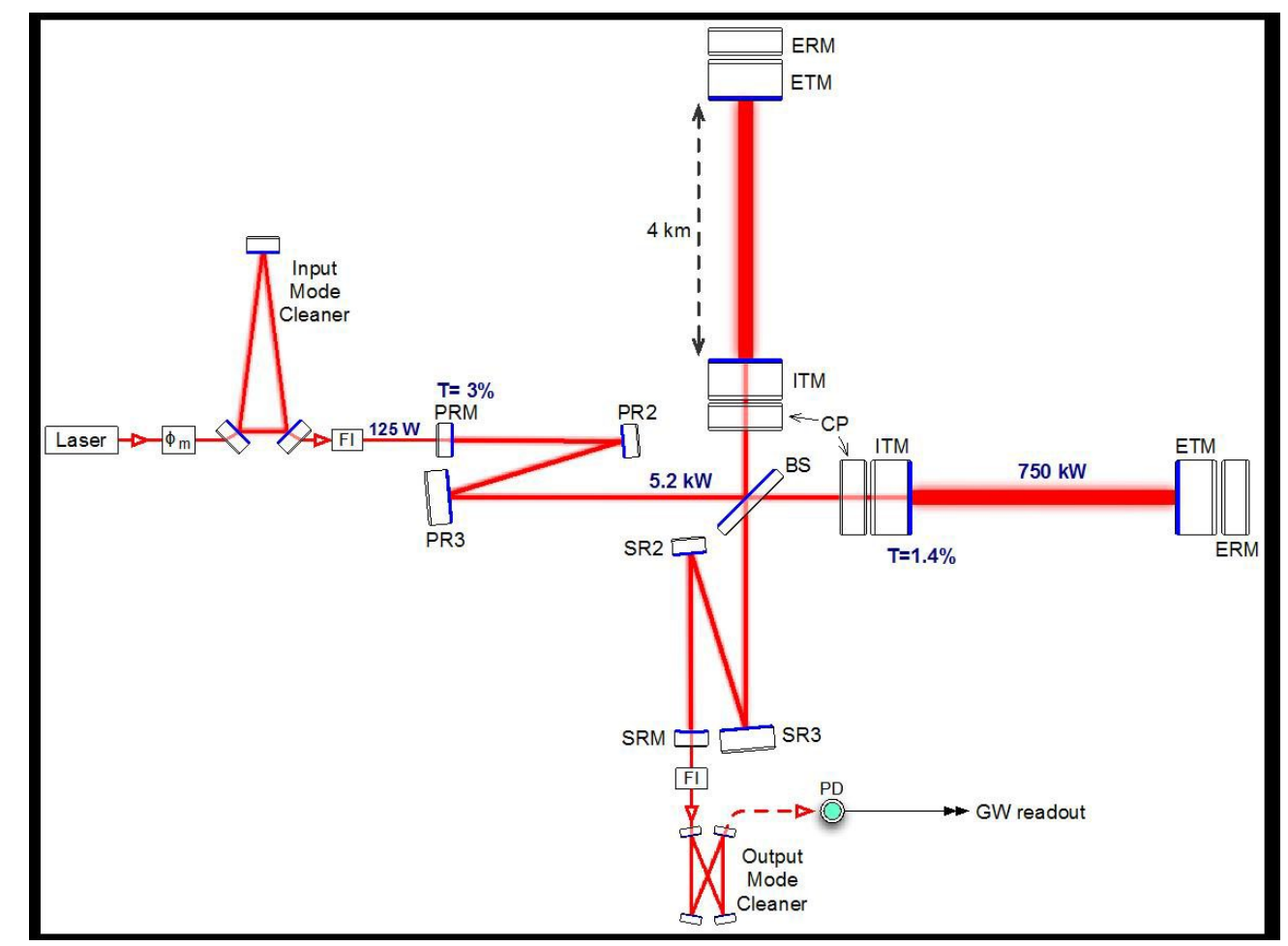

Figure 1. Advanced LIGO optical configuration. ITM: input test mass; ETM: end test mass; ERM: end reaction mass; CP: compensation plate; PRM: power recycling mirror; PR2/PR3: power recycling mirror 2/3; BS: 50/50 beamsplitter; SRM: signal recycling mirror; SR2/SR3: signal recycling mirror 2/3; FI: Faraday isolator; $\varphi$ m: phase modulator; PD: photodetector. The laser power numbers correspond to full power operation. All of the components shown, except the laser and phase modulator, are inside the LIGO UHV system. 
Table 1 - Main parameters of the Advanced LIGO interferometers [6].

\begin{tabular}{cc}
\hline Parameter & Value \\
\hline Arm cavity length & $3994.5 \mathrm{~m}$ \\
Arm cavity finesse & 450 \\
Laser type and wavelength & Nd:YAG, $\lambda=1064$ \\
Input power, at Power Recycling Mirror & Up to $125 \mathrm{~W}$ \\
Beam polarization & Linear, horizontal \\
Test mass material & Fused silica \\
Test mass size \& mass & diameter x $20 \mathrm{~cm}, 40 \mathrm{~kg}$ \\
Beam radius (1/e $e^{2}$, Input Test Mass (ITM) / End Test & $5.3 \mathrm{~cm} / 5.2 \mathrm{~cm}$ \\
Radius of curvature, ITM / ETM & $1934 \mathrm{~m} / 2245 \mathrm{~m}$ \\
Input mode cleaner length \& finesse & $32.9 \mathrm{~m}$ (round trip), 500 \\
Recycling cavity lengths, Power Recycling Cavity / & $57.6 \mathrm{~m} / 56.0 \mathrm{~m}$ \\
\hline
\end{tabular}

\section{WHY LIGO CARES ABOUT CLEANLINESS}

LIGO utilizes a 200W Nd:YAG continuous wave laser. The peak fluence at high power will be $96 \mathrm{~W} / \mathrm{mm}^{2}$ for the larger optics (ETM and ITM optics in Figure 1) and up to $2000 \mathrm{~W} / \mathrm{mm}^{2}$ on some of the smaller optics (input mode cleaner optics in Figure 1). During operation of the interferometers, even low-level contaminants can induce pin-hole damage in the optic coatings, which would in turn affect the overall detector sensitivity. Even with stringent cleanliness protocols in place, higher than anticipated levels of contamination were found in assembly areas and inside vacuum chambers at both observatories. An example of this is highlighted in Figure 2. Installation teams reported:

- Plumes of new particles generated by chamber activity.

- Concentrations of $>1-5$ particles/cubic foot persisting tens of minutes.

- Numerous small and large (D $>100 \mu \mathrm{m})$ "acquired" particles on suspended optics.

- High density of stainless steel particles on interior vacuum chamber walls.

- Pump-down and vent cycles lofting in-chamber particles.

In response, a massive effort was made to quickly quantify the extent of contamination, identify its sources, improve cleanroom practices to decrease the generation of particulates, and develop non-contact cleaning methods for installed optics in air. The most common specimens found during this effort were metals [12], which is not surprising considering that the majority of the contents of LIGO's UHV system are tens of thousands of bolted together metal parts. It should be pointed out that the total quantity of biological specimens found as part of this effort is misleadingly low as with the exception of hair, biological samples tend to be very small and are often overlooked. Analysts within LIGO have found that there is a tendency to focus analysis on larger particulates ( $20 \mu \mathrm{m}$ and greater) because they are easier to find and easier to get good readings from. However, from this work it was clear that there is no smoking gun, i.e. no single source of particulate contamination. LIGO's cleanliness requirements for optics, and the Contamination Control (CC) protocols required through all phases of work to meet those requirements are described in detail in the subsequent sections. 


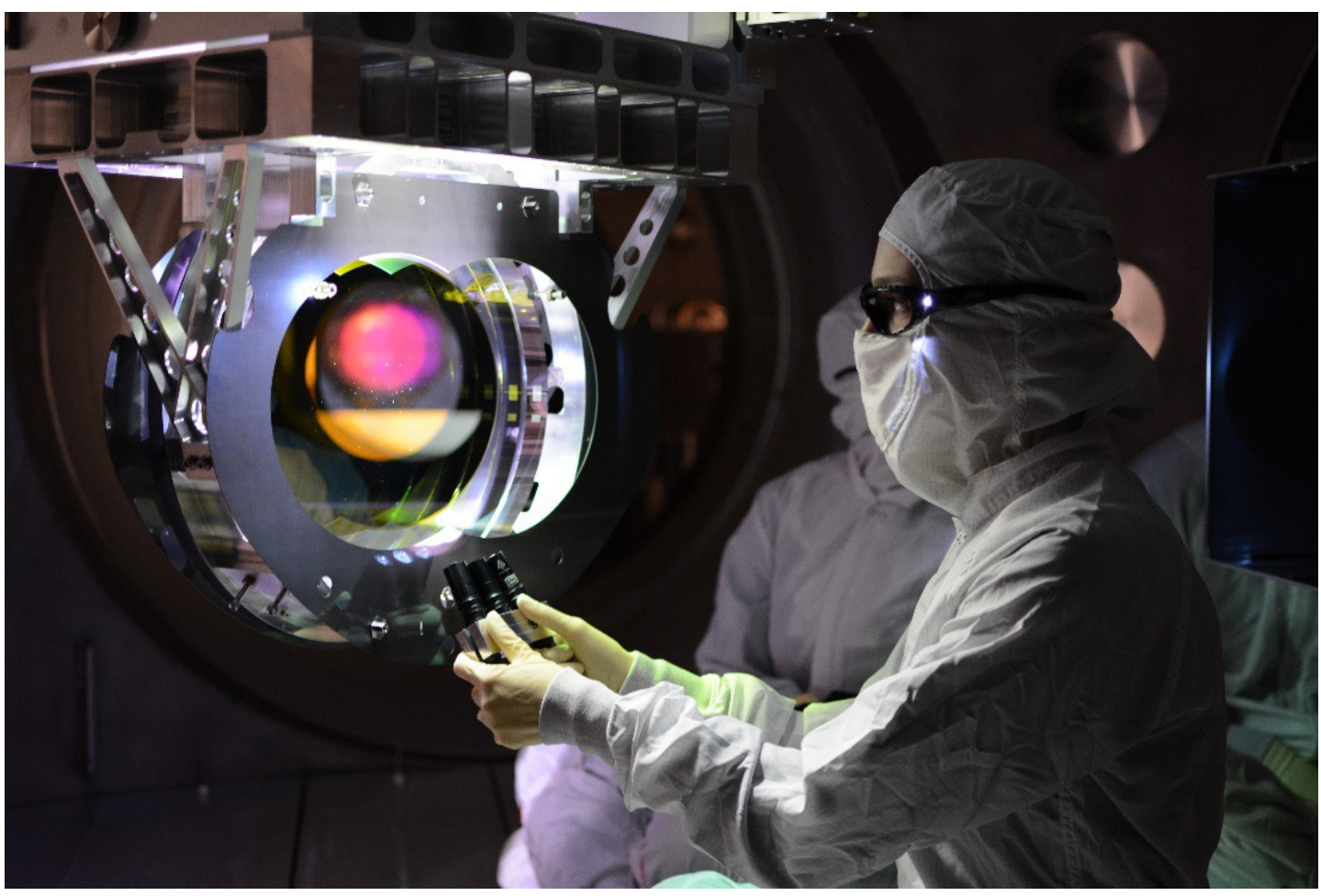

Figure 2. Particulate contamination on LLO beamsplitter (BS), see Figure 1, is visible from across the chamber

\section{PARTICULATE CONTAMINATION REQUIREMENTS}

Surface particles absorb and scatter power from the interferometer mode. For Advanced LIGO, the absorption requirements $(\leq 0.1 \mathrm{ppm})$ are more restrictive than scattering requirements $(\leq 5 \mathrm{ppm})$ for allowable particulate contamination [13]. The requirements for the large fused silica optics are summarized in Table 2 [14].

Table 2. Absorption requirements for large aLIGO optics.

\begin{tabular}{|l|l|}
\hline Total Cumulative HR Surface Absorption [15] & $<\mathbf{1 ~ p p m}$ \\
\hline Measurements of as-built coating absorption & $\mathbf{0 . 3} \mathbf{~ p p m}$ - typical \\
& $\mathbf{0 . 4} \mathbf{~ p p m}-\mathbf{~ m a x}$ \\
\hline Allowable absorption due to contamination & $\mathbf{0 . 7} \mathbf{~ p p m}$ \\
Hydrocarbon film deposition & $0.1 \mathrm{ppm}$ \\
Particulates & $0.6 \mathrm{ppm}$ \\
\hline
\end{tabular}

The $0.6 \mathrm{ppm}$ absorption due to particulate contamination translates to a surface cleanliness level requirement of 65 [14], (per IEST-STD-CC1246D) [16]. This assumes a spherical particle, with an emissivity of 1, is centered on the optic within a $\sim 60 \mathrm{~mm}$ beam with a Gaussian intensity distribution. Although strict, the requirement is comparable to cleanliness levels achieved by other projects using similar contamination control approaches. For example, the National Ignition Facility (NIF) requires cleanliness levels of 50 and 100 for its large and small optics, respectively [17]. NASA's Goddard Space Flight Center measured a cleanliness level of 35 for optics on the Solar Terrestrial Relations Observatory (STEREO) spacecraft [18], and the Jet Propulsion Laboratory measured and reported a cleanliness level of 92 for the Mars Science Laboratory rover [19]. 


\section{CONTAMINATION CONTROL AND MITIGATION}

A Contamination Control Working Group (CCWG) was formed and charged with collecting, characterizing, and analyzing contamination in the vacuum system, as well as researching and implementing solutions. The CCWG took a holistic approach to evaluate the contamination problem, and develop effective mitigation techniques. All the potential contributing factors were considered, and nothing was taken for granted or assumed to be unimportant. Room for improvement was found in unexpected places. Successes, challenges, and changes to LIGO's practices are discussed.

\subsection{Facilities and Cleanrooms}

All assembly and installation work is performed in ISO 5 (Class 100) cleanrooms, and the main areas surrounding these cleanrooms are ISO 7.8 (Class 50,000) [20]. How and when to clean the cleanrooms themselves is a fundamental, though often overlooked, aspect of contamination control. The floors are typically the most contaminated part of a cleanroom, as many particulates are tracked in on feet and airborne contaminants eventually settle on the floor. Without routine cleaning, the floor level contamination will be stirred back up or redistributed by foot traffic and work activities. A clean cleanroom starts with a clean floor. How to clean a floor seems incredibly straightforward, until the CCWG found that LIGO's method was less than optimal. A large number of witness samples contained a complex mix of silicates, identified in industry as the result of foot traffic [21]. The majority of LIGO's clean areas have vinyl flooring over concrete; however, in some areas, there is only a sealed concrete floor. Both types of flooring are vacuumed, and then mopped. This is done daily for critical areas, such as around an open vacuum chamber; and $2-3$ times a week for all other areas, such as clean areas around critical cleanrooms and the bake facility. Upon investigation, the cleaning solution and floor cleaning equipment were found lacking. A mixture of $5-10 \%$ isopropanol in water - hot, not cold water, is now used. To improve the efficacy of mopping and standardize protocols across all of LIGO, each observatory implemented new $100 \%$ polyester knitted mop heads (Contec ${ }^{\circledR}$ Inc. \#ELMOP) and double bucket system (Contec ${ }^{\circledR}$ Inc. \#2720), which allows for differentiation between cleaning and rinsing solutions. It has been found that vacuuming followed by mopping with this cleaning mixture in combination with the double buckets is effective at reducing particulate contamination and is also non-invasive [22].

The cleaning crew has been invaluable in identifying cleanliness issues and adapting to correct them. One such issue was LIGO's narrowly focused approach to contamination control. After spending time on the floor with the cleaning crew, it became obvious that control policies had to be extended beyond critical areas to mitigate contamination before it became a problem. One example is the cleanliness and layout of the facilities leading up to the vacuum envelope. The transition area, the region between the main office suites and initial clean areas, had been largely ignored. All foot traffic and carts pass through the transition area, and as a result, it had a massive impact on the cleanliness of the subsequent clean areas. The configuration of the transition area entrance was optimized, and now includes an area for washing dedicated cleanroom shoes, gross cleaning station, and new signage. Adjusting the position of a bench created a more obvious definition between the dirty side and clean side where shoe covers are required, thus reducing cross-contamination. These changes required little to no cost, but had a significant impact.

Removable sections of flooring designed to combat particulate contamination, shown in Figure 3, are utilized to further control floor level contamination in critical areas (Dycem CZ01 Cleanzone). Clutter in and around cleanrooms is cleared to support gross cleaning. Non-essential areas and bulky items near the chamber, such as cable trays, racks of electronics and external isolation systems, are wiped down and then wrapped in clean plastic sheeting. Instead of settling into nooks and crannies that are difficult to reach and clean, airborne particles settle on the angled plastic sheets; making them easy to spot and remove. Additional cross-flow or draw fans are placed adjacent to work areas to augment the existing fans and filters within the cleanrooms. Following all of these changes, the cleaning crew reported a dramatic improvement in the cleanliness of used wipes and shoe covers.

Maintaining the cleanliness of the cleanrooms is arguably the most inexpensive and effective way to protect the optics. It requires commitment from everyone, not just the cleaning crew, because everyone contributes to the problem. 

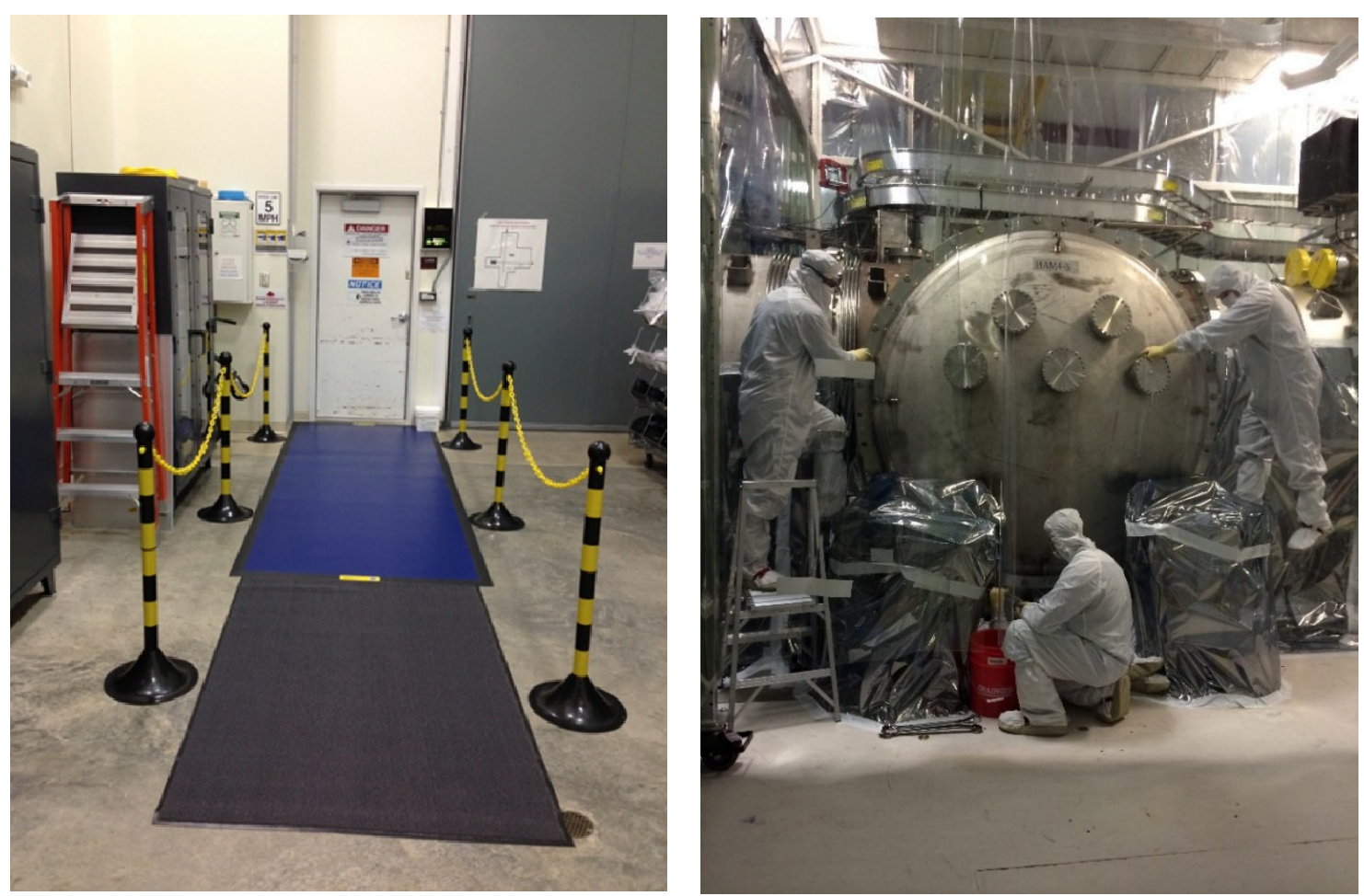

Figure 3. Dycem flooring in transition area, a high traffic area (LHS).

Figure 4. Equipment outside the chamber wrapped with clean plastic sheeting.
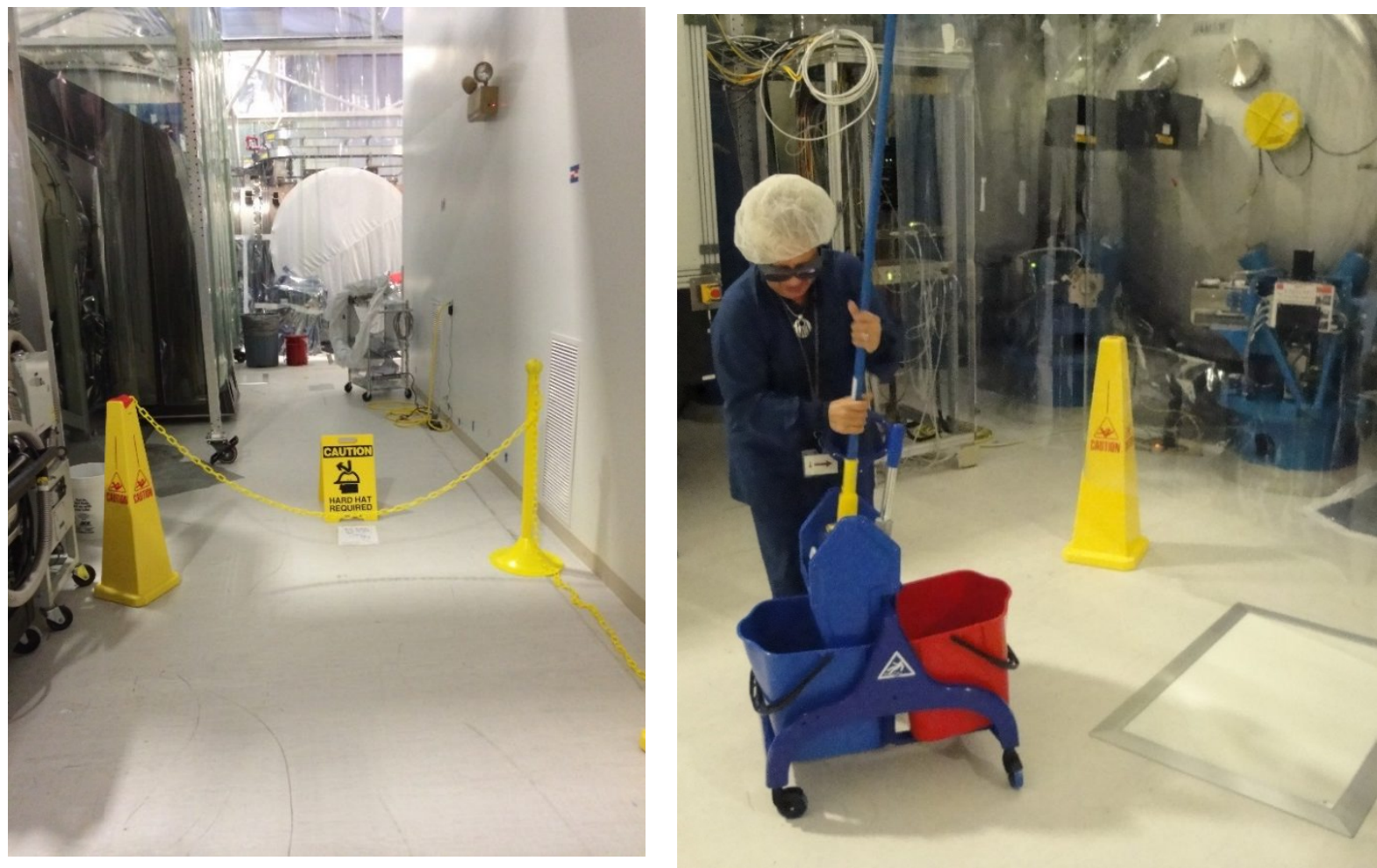

Figure 5. Barriers and signs placed during chamber closeout activity to deter foot traffic (LHS).

Figure 6. Double bucket in use by one of the cleaning staff. 


\subsection{Preparing parts for UHV service}

To limit outgassing, each part in service within the interferometers is comprised solely of materials and components that have been approved for use in the LIGO UHV system. The majority of parts were machined from stainless steel (304 SSTL) and aluminum (Type 6061-T6) [23]. Final surfaces of metal components were machined to a maximum surface roughness of 63 micro-inch $(\mathrm{Ra})$ [24]. Every single individual part was cleaned and baked in accordance with the LIGO specifications [25]. The default bake-out procedure for parts and assemblies is vacuum baking. Vacuum bake loads generally consist of parts made from the same material, so as not to compromise the cleanliness of parts by baking at the lowest temperature defined for the materials in a mixed load. Stainless steel loads are baked at $200^{\circ} \mathrm{C}$ for 48 hours. Due to the fact that tempered aluminum is very sensitive to reheating, and can lose significant yield strength with excessive bake temperature or time, aluminum (Type 6061-T6) loads are baked at $150^{\circ} \mathrm{C}$ for 48 hours while all other types of aluminum used are baked at $120^{\circ} \mathrm{C}$ for 48 hours. Using the time and temperature cycles quoted kept strength losses (in aluminum) to less than 5\% [26]. After clean and bake, parts were tested to confirm compliance with surface cleanliness requirements [25]. It is essential to maintain these levels of cleanliness through the subsequent processes of packaging, storing, assembly, testing, transportation, installation and commissioning.

\subsection{Wrap, Bag and Tag}

After cleaning, all parts are wrapped, bagged, and tagged [26]. Because once is not enough, parts are double wrapped in foil (All Foils Inc. UHV Foil) that meets ASTMB 479 [27], and then double bagged in silver anti-static foil/poly ISO 5 (Class 100) zipper barrier bags (Seco Pack \#512). At all stages, assemblies must be covered with C3 static-dissipated fabric covers (Burlington C3) within the cleanroom when not in use. Clean stainless steel containers are also used to cover and store tools, spare parts, and smaller assemblies, when not in use.

\subsection{Storage}

Some LIGO parts are being placed into long term storage ( $~ 5$ years) rather than being installed into the UHV system. The majority of the metal parts that are stored in this way are placed into clean storage containers which have their temperatures controlled to $72 \mathrm{~F}+/-3.5 \mathrm{~F}$ and relative humidity controlled to $<10 \% \mathrm{RH}$. This is achieved using desiccant units with molecular sieve technology [28] which meets MIL-D-3464E, Type II (non-dusting) [29]. Critical components (e.g. silver-coated optics) are stored at $<4 \% \mathrm{RH}$ with dry nitrogen replacing the desiccant [30].

\subsection{Assembly}

While there are some welded parts in the LIGO UHV system, the majority of parts are bolted together with socket head cap screws. Tests were conducted to evaluate the tendency to gall and to collect particles generated by screw threads that were thoroughly cleaned for UHV service. Bars of stainless steel and aluminum were tapped to size 1/4-20 using a wide variety of tap configurations, with 20 holes per bar. Strips of transparent tape were placed under the row of holes in each bar. On half of those holes, screws of various materials and treatments were inserted, torqued, and removed 10 times per hole to check for the effects of material and tap choices on galling tendencies. Generated particles were collected by the tape, which was transferred to a report sheet and scanned [31]. Based on these tests, the following recommendations were put in place for all parts used in the LIGO UHV system:

- Aluminum - LIGO aluminum in-vacuum parts that are expected to be disassembled (through phases of assembly and install) must use stainless steel screws in Nitronic-60 (N60) thread inserts for the tapped holes, to reduce the amount of generated particles and to reduce the risk of galling. For parts that are disassembled rarely, stainless steel screws shall be used in 0.005 " oversize tapped aluminum parts. Silver plated screws should not be used in aluminum threaded holes as they are likely to cause galling (cold welding).

- Stainless Steel - Silver-plated, stainless steel screws shall be used in 0.005" oversize tapped stainless parts [32].

It should be noted that in addition to rigorous checks during manufacture via Quality Assurance (QA) processes, Go-NoGo Gages were available at all assembly stations along with Allen keys and heli-coil insertion tools to check holes prior to use.

\subsection{Cleanroom Garb}

One of the largest sources of contamination comes from the people working in a cleanroom. Body box testing was used to evaluate particle generation by humans and their clothing, and the particle generation and filtration efficacy of existing 
LIGO garb [33]. Several LIGO engineers were used as test subjects. Each subject performed a series of defined movements in a small ISO 3 cleanroom, and the number and size of particles emitted were recorded. Brand new and old cleanroom suits were worn over a variety of street clothes (synthetic vs. natural fibers, shorts vs. long pants) and medical style scrubs made from low particulating ESD micro denier material (Worklon \#1920 \& \#1930). Given the high variation in particle generation between individuals, relative performance for each engineer was compared.

Newer garb proved better at shielding contamination from test subjects and their street clothes, but generated more particles than old garb. Wearing scrubs instead of street clothes under existing LIGO garb offered at least $1 / 2$ class improvement in cleanroom class (factor of $\sim 3$ reduction in particles). The majority of the testers were an entire class cleaner when wearing scrubs [33]. As a result of the body box tests, the life of the garb already in service was extended and particulate contamination was mitigated by introducing scrubs [34]. All LIGO personnel working in a cleanroom, from the cleaning crew to visiting scientists, are required to wear scrubs instead of street clothes under cleanroom suits. While the cleanroom suits are professionally cleaned on a regular basis and stored in common areas for use by all, scrubs are given to individuals and are laundered at home. The policy change had intangible benefits as well. The introduction of scrubs drew attention to the cleanliness problem. The sea of blue uniforms every morning is a perpetual reminder that everyone impacts, and is therefore responsible for contamination control.
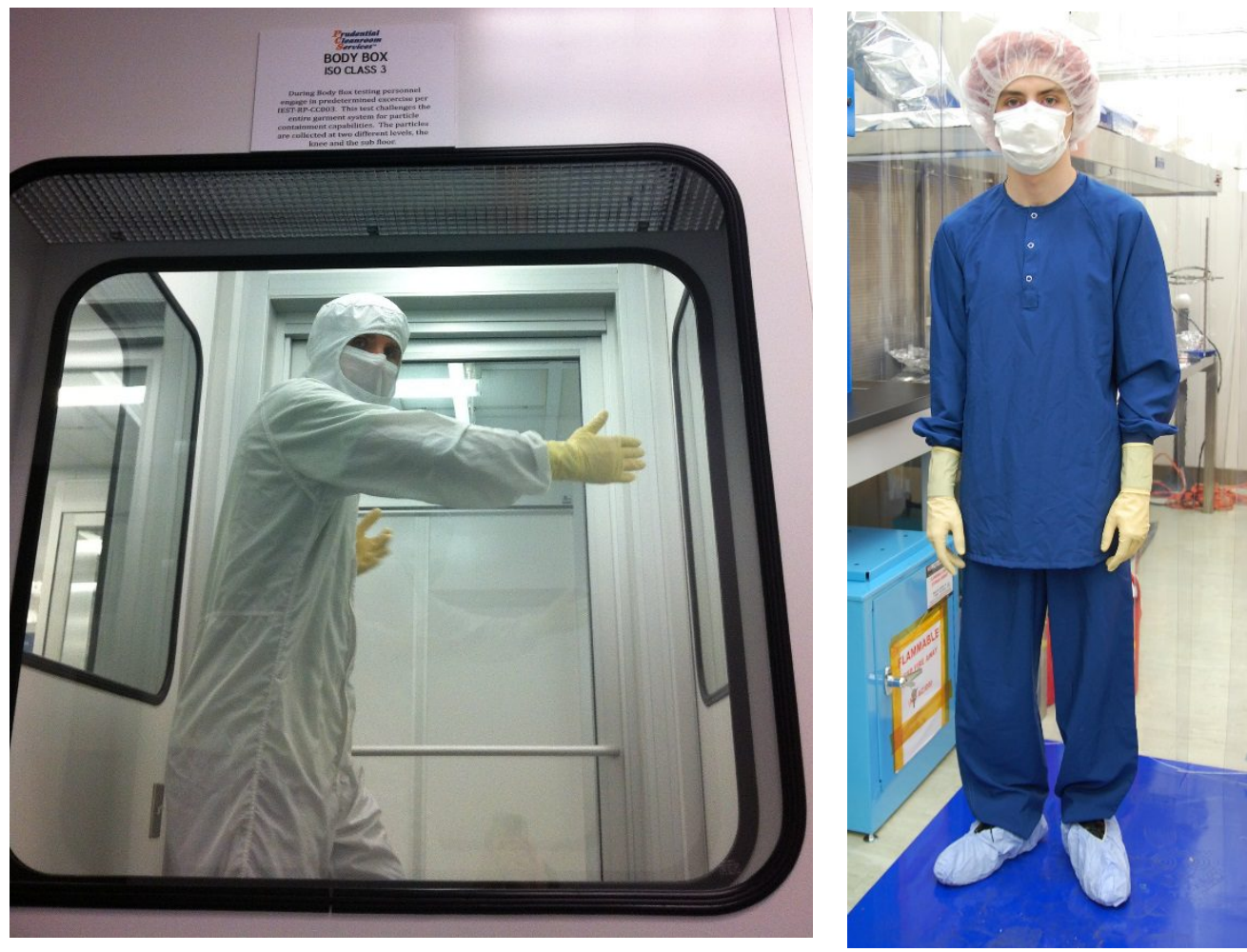

Figure 7. Body Box testing of cleanroom garb (LHS).

Figure 8. LIGO person in Scrubs, made from ESD micro denier material (Worklon \#1920 \& \#1930).

\subsection{Cleanroom Gloves}

Because UHV gloves are the most common "tool" in contact with clean surfaces, maintaining their cleanliness is critical to successful contamination control. LIGO-approved UHV latex gloves (Ansell AccuTech Ultra-Clean Latex 91-300) come in a variety of sizes and are hand specific to aid with dexterity for fine assembly work. Two sets of gloves are worn, often over glove liners, to ensure multiple layers of protection between Class A parts and human skin. After donning outer gloves, and prior to contact with clean surfaces, it is imperative to wash the gloves with a wipe presaturated with 100\% isopropyl alcohol (Texwipe TX8410). Gloves must be allowed to dry before starting work. This is easily verified by eye and requires little time. If one becomes "dirty," the same method is used to wash the gloves to 
become "clean" again. This is done instead of replacing the outer gloves per the original LIGO protocol. Gloves should be replaced if they rip or become contaminated beyond what can realistically be removed by wipes. Tests have shown that cleaning the gloves prior to use makes a significant improvement to the cleanliness of the gloves:

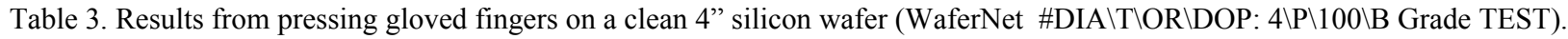

\begin{tabular}{|l|l|l|l|}
\hline & $\begin{array}{l}\text { Control (blank section of } \\
\text { wafer) }\end{array}$ & $\begin{array}{l}\text { Fingers press with } \\
\text { gloved hand using gloves } \\
\text { fresh out of the bag }\end{array}$ & $\begin{array}{l}\text { Finger press with gloved } \\
\text { hand after cleaning with } \\
\text { wipe (as described above) }\end{array}$ \\
\hline $\begin{array}{l}\text { Particles collected } \\
\text { counted }\end{array}$ & 2 particles $/ \mathrm{cm}^{2}$ & 100 's particles $/ \mathrm{cm}^{2}$ & $<10$ particles $/ \mathrm{cm}^{2}$ \\
\hline $\begin{array}{l}\text { Size range of particles } \\
\text { collected / counted }\end{array}$ & $\begin{array}{l}\text { Particles in size range } \sim 20 \\
\mu \mathrm{m}\end{array}$ & \\
\hline
\end{tabular}
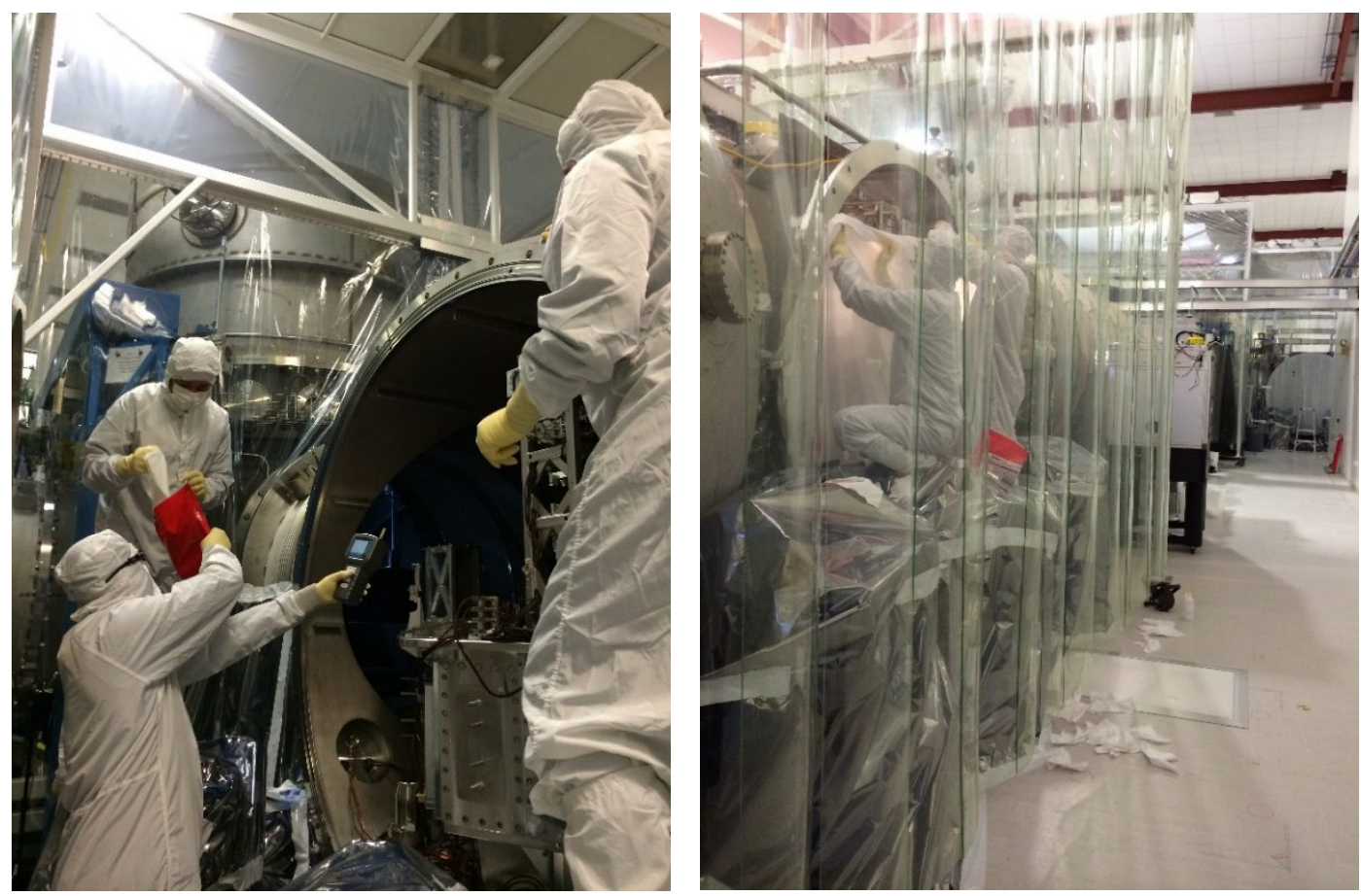

Figure 9. Engineer takes a particle count while passing around pre-saturated wipes for washing gloves (LHS).

Figure 10. Engineers accessing on of the UHV chambers by removing the temporary $\mathrm{C} 3$ soft door cover.

\subsection{Clean As You Go policy}

A great deal of contamination is generated during assembly and installation processes performed by the assembly crews [35]. Furthermore, it is impossible to eliminate all sources of contamination entirely, regardless of the protocols and equipment in place. Therefore, a "Clean As You Go" policy must become part of daily cleanroom practices. "Clean as you go" is what it sounds like - regularly and consistently cleaning before, during, and after work; and applies to everyone working in a cleanroom. The following basic tools are an integral part of the policy:

a) Handheld Tiger vacuum cleaners (Tiger-Vac, $M V-1 C R$ (BP) Series) both in cleanrooms and in chambers. If areas are accessible to vacuum cleaners, one should always vacuum before wiping. 
b) An excellent method for removing dust from surfaces, which is now being used across LIGO, is wet wiping with the same pre-saturated wipes used for glove washing. The best technique to efficiently remove contaminants is to wipe from top to bottom and in linear, overlapping strokes. Circular wiping redistributes contaminants over the area just cleaned, and is therefore unacceptable. In addition, one should refold wipes to expose a fresh wipe surface for each run. Dry wipes or wipes manually soaked with $99.5 \%$ isopropanol (VWR ACS Grade BDH1133-1LP) are also used depending on the application. For hard to reach areas, swabs (Absorbond Series Swabs TX762) have proven highly effective.

c) High intensity 215 lumen lights are used at grazing incident angle to illuminate particulates on components, assemblies and optics tables. Three flashlights (Fenix LD22) are housed in a machined array [36] aligned to produce an uninterrupted 4 " beam.

d) Particle counters are used to measure airborne particles (Met One HHPC $3+\& 6+$ ). The levels detected from this device can not only indicate whether a problem exists within the clean space, but can also determine whether or not work re-starts following a cleaning period, or if more time is required for the particulates to settle [37].

Measurements are made by teams before work starts in a cleanroom or in a chamber within a cleanroom. Typically counts (measured in counts $/ \mathrm{ft}^{3}$ ) at this point are $<30$ for $0.3 \mu \mathrm{m}$ and $<2$ for $1.0 \mu \mathrm{m}$. Typical numbers recorded in chamber (following 3 hours) in-chamber work by 1 person (and before any cleaning) range between 700-1300 counts for $0.3 \mu \mathrm{m}$ and 10-300 counts for $1.0 \mu \mathrm{m}$. The user typically stops work for 5 minutes before taking these readings. Under these conditions the counts return to pre-work levels within 10-30 minutes, although it can take longer and users usually continue to monitor airborne particles during this period. During cleaning periods, such as vacuuming, counts are typically 3000 for $0.3 \mu \mathrm{m}$ and 650 for $1.0 \mu \mathrm{m}$ with a settling time of $2-3$ hours. Iterative cleaning is the key in removing the overall dust load and consequently the amounts stirred up from each work session.

e) Installation crews utilize $35 \mathrm{~W}$ UV-A inspection black light lamps in a survey manner to highlight areas where particulate contamination may not be immediately obvious. Tests have shown that there is no risk using this light in proximity of LIGO coated optics [38], however direct illumination of key optical surfaces is not recommended. All personnel working with the UV black light flashlights wear protective safety eyewear, as demonstrated in Figure 11[39].

During all stages of clean assembly, including at the end of assembly (ahead of storage, transport or installation), it is essential to periodically: -

(i) Inspect components with the visible light (flashlight array).

(ii) Vacuum followed by wipe/swab or wipe only depending on access.

(iii) (The UV-A black light should be on hand to use if contaminants are found or suspected. )

(iv) Check the airborne particle levels with a hand-held particle counter.
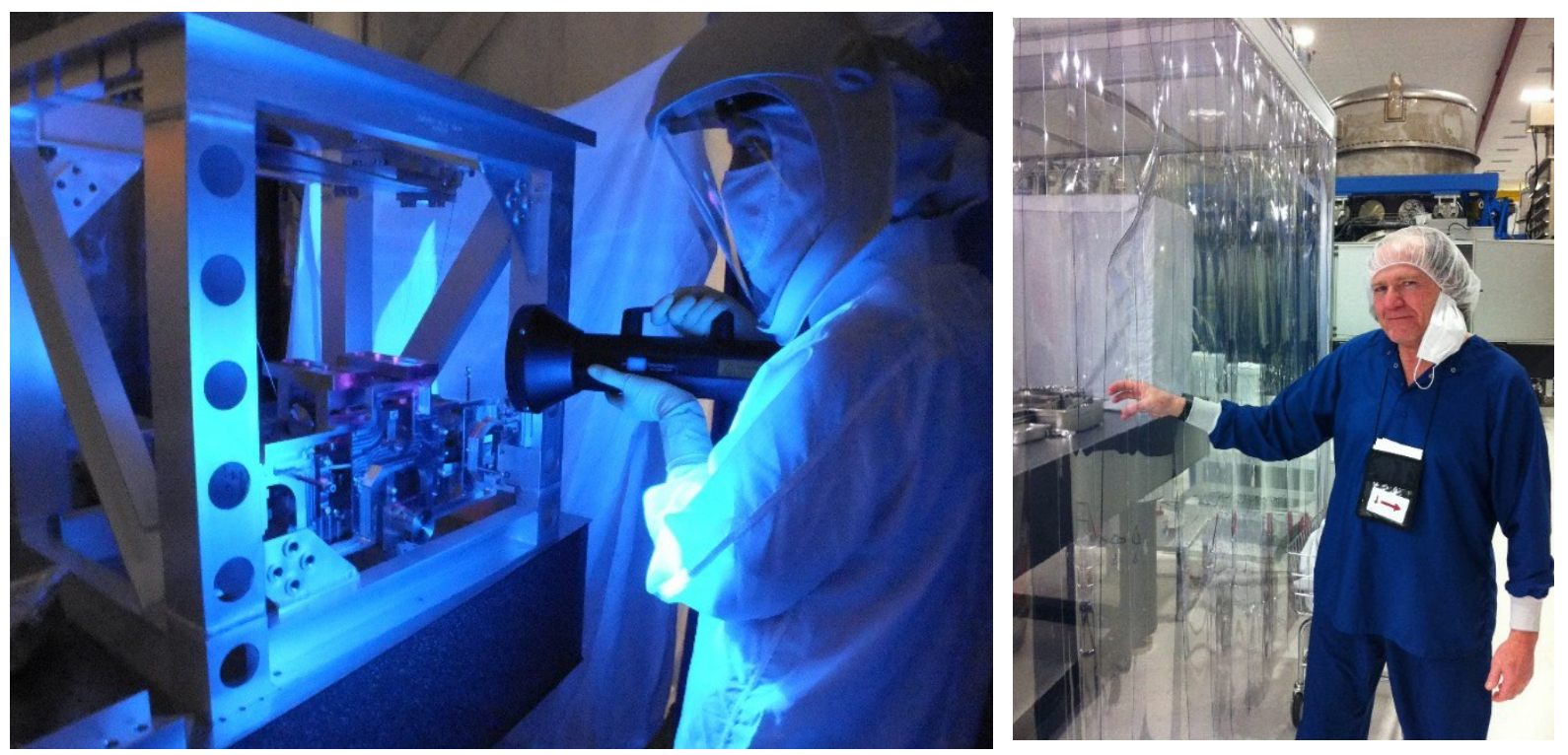

Figure 11. Black light inspection of assembly (LHS).

Figure 12. LHO Contamination Control lead demonstrates what not to do in a cleanroom. Images of common mistakes were featured in an all-hands meeting on contamination control [12]. 


\subsection{Training}

Working in a cleanroom gives a false sense of security with respect to cleanliness, and it is easy to become complacent. A fundamental aspect of the contamination control is training. The CCWG introduced a suite of training materials, which were split into two levels [40]. Level I is required for all workers, from visitors to the cleaning crew to senior engineers. Level II has a number of sub-categories for specialty workers, including those operating in the vacuum chambers. There is also training material and approved buy lists for the business office to ensure consistency in purchases across multiple sites.

Work inside cleanrooms is controlled through a work-permit process, and access to cleanrooms and surrounding areas is restricted. New visitors, including visiting LIGO staff, must complete contamination control training and pass a test before being granted badge access to clean areas; and refresher training was required for all on-site staff. A Contamination Control lead was appointed at each observatory, and tasked with monitoring cleanliness and facilitating contamination mitigation. Like laser safety, noncompliance with protocols has the potential to do serious harm. As a result, the lead may require individuals to repeat training or restrict their badge access if necessary.

\subsection{Protection of Optical Surfaces}

Optical surfaces were protected whenever possible during assembly, testing, and installation. This is achieved utilizing lens caps, metal shields (dust barriers), and/or a protective layer of First Contact Polymer ${ }^{\text {TM }}$ (FC). When key optical surfaces are exposed a last lick cleaning utilizing the FC is performed. FC is summarized in more detail in the next section of this paper.

\subsection{First Contact ${ }^{\mathrm{TM}}(\mathrm{FC})$ application}

First Contact ${ }^{\mathrm{TM}}$ (FC) Polymer (Photonic Cleaning \#RFCF) is a mixture of solvent and polymer that is applied in liquid form to an optical surface, let dry, and then removed to leave a clean optic. Over the last few years LIGO has subjected FC to a battery of tests before qualifying it for use. A full contingent of documents outlining tests, procedures, and designs are available on LIGO's online Document Control Center [41]. Due to concerns that the mechanical action of brushing on FC may drag contaminates across the surface hard enough to scratch the delicate coatings, LIGO developed a non-contact application method to provide last lick cleaning of optical surfaces prior to closing the vacuum chambers [42].

A conical fixture used in conjunction with a spray mixture provides a simple, clean, precise, repeatable method for cleaning optics. The fixture is bolted onto a suspension, and the High-Density Polyethylene cone is gently slid against the face optic [43]. An intricately folded wipe secured to the end of the cone creates a seal between the optic and cone. A 3 oz HDPE bottle and atomizing nozzle is filled with a 1:1 ratio of red FC to red FC thinner (Photonic Cleaning \#RFCF $\& \# R T F C F$ ). This optimal mixture lowers the polymer content enough to avoid cobwebbing in the dry vacuum chamber. The polymer mix is sprayed onto the circular area of the optic marked by the cone, and allowed to dry. Excess polymer is contained by the cone walls and absorbed into the wipes. After spraying and allowing three layers to dry, the cone is removed. The outer edge of the sprayed FC must be thickened so that the entire film can be pulled off in one piece. A "pie crust" is painted over the edge of the sprayed FC circle using a brush (Gordon Brush Mfg. Co. Inc. custom order $3 / 8$ " natural tapered with nylon filament.). The painted section is well outside the beam area. This method yields uniform and consistently sized films of repeatable diameter, protects the optic from overspray as well as offering protection to the surrounding area from the airborne polymer [42].

When the FC spraying is done in an enclosed area, LIGO operators wear protective masks (3M 6100/07024 respirator with $3 M 60923$ cartridges). If overspray from the FC should contaminate an optic in a non-critical location, such as the barrel, it is removed with a folded lens wipe (Berkshire Lens X 90) soaked in acetone. If further information is required, please contact the authors of this paper.

\subsection{First Contact ${ }^{\mathrm{TM}}$ (FC) removal}

Once dry, the FC film is peeled with the aid of a Victrex ${ }^{\circledR}$ PEEK ${ }^{T M}$ mesh tab (McMaster-Carr \#9289T11). Even if the area around the optic appears clear, it is critical to remove charge on the optic induced by peeling the dry FC to prevent the attraction of airborne contaminants to the freshly cleaned surface. Nitrogen gas is blown onto the optic from an ionization system that emits a stream of balanced ions (Simco-Ion Model Top Gun ${ }^{T M}$ ). Due to evidence of 1 - $5 \mu \mathrm{m}$ particles emitted by the system at higher pressures, the gas flow in the Ionizing System is restricted at $10 \mathrm{psi}$ (when used in conjunction with FC removal from LIGO optics). Tests have shown that in order to remove all residual charge, it is 
essential to continue with the ionization of the surface for 4.5 minutes per optic surface. Measurements of charge are made periodically with an electrometer (AlphaLab Inc. \#VSVM).

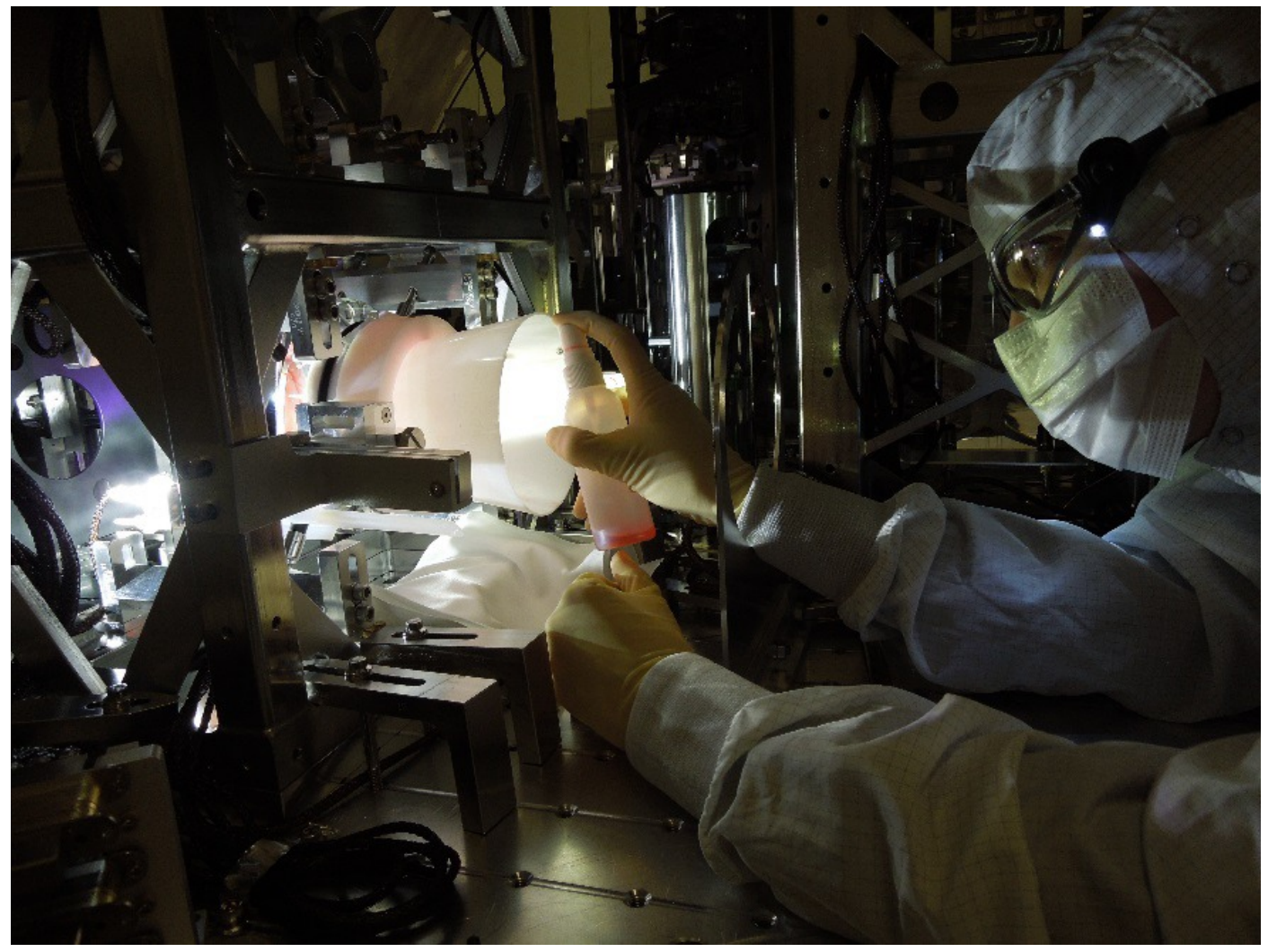

Figure 13. Spray application of First Contact on an Input Mode Cleaner optic.
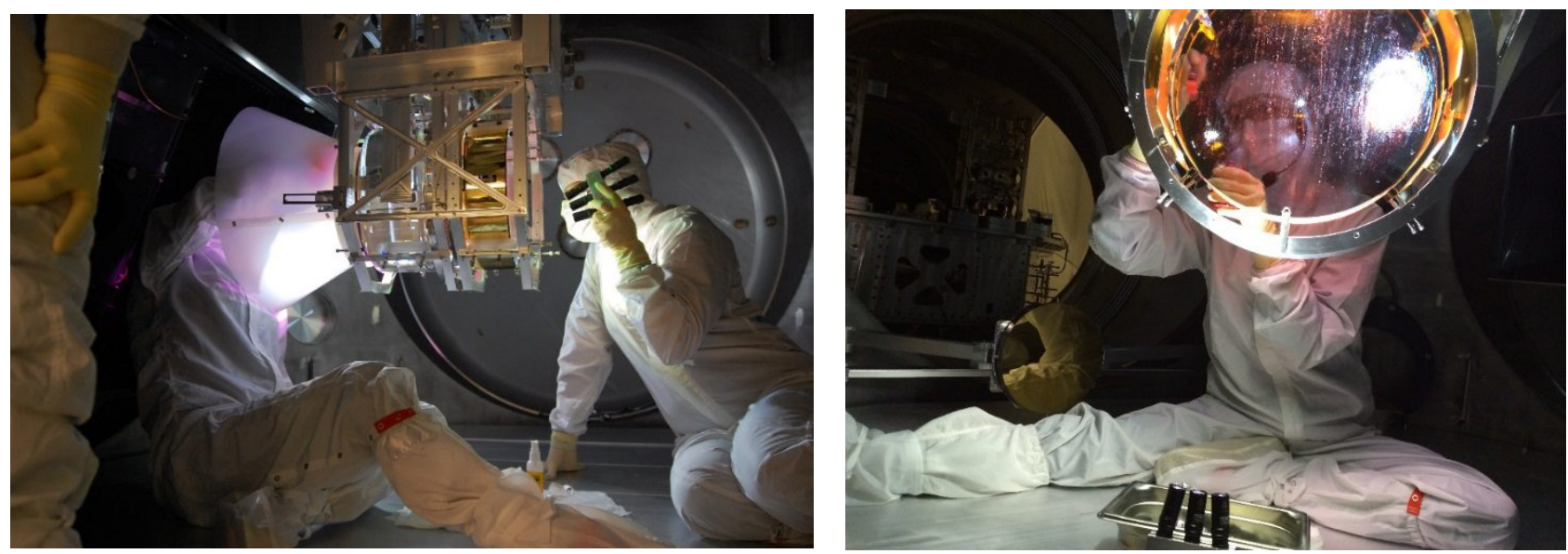

Figure 14. Spray application of First Contact on a large ITM optic (LHS).

Figure 15. Painting a crust of First Contact around the sprayed First Contact on one of the large optics, one side of the beamsplitter optic (BS), see Figure 1. 


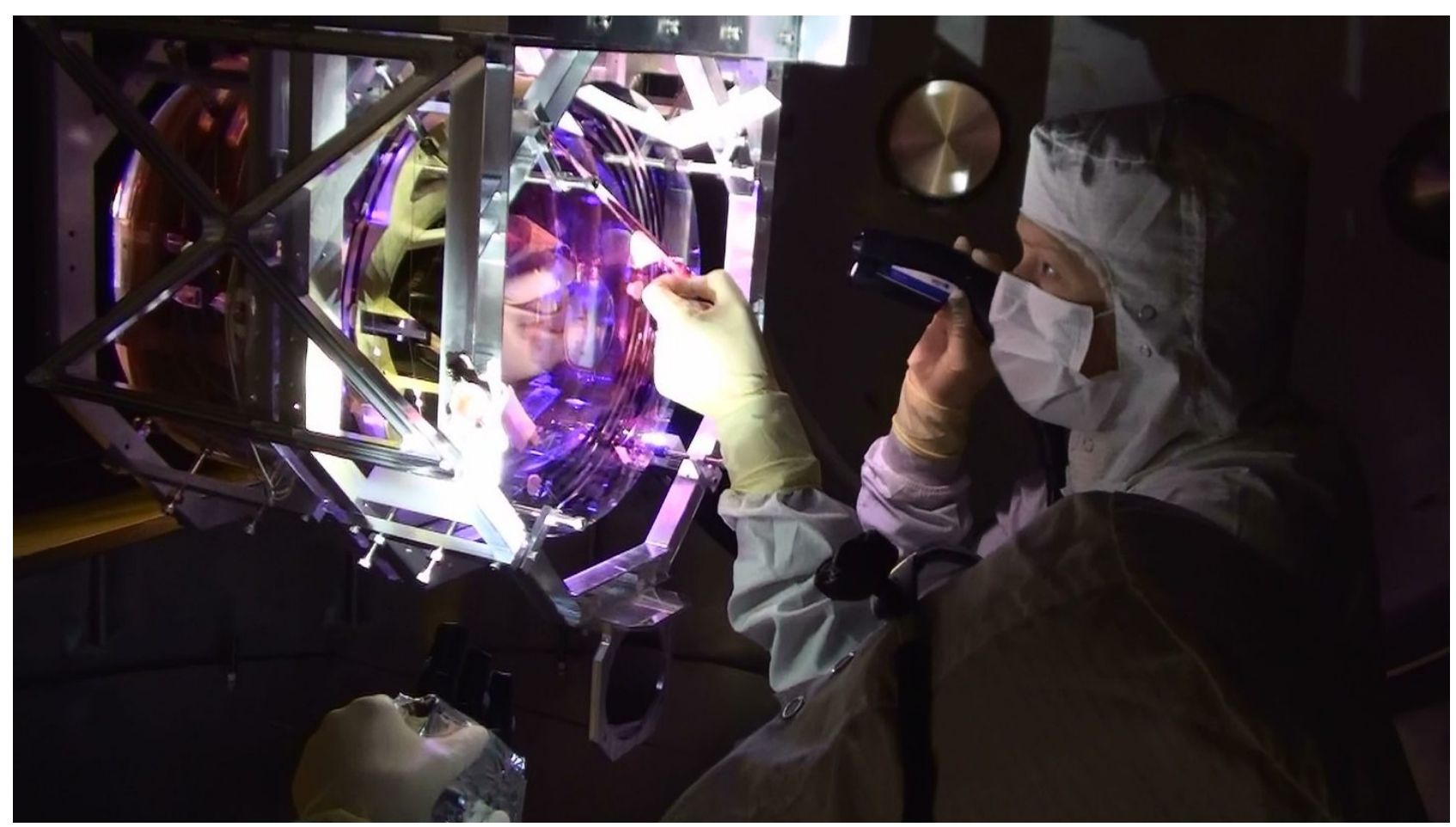

Figure 16. Peeling First Contact from a large ITM optic.

\section{RESULTING CLEANLINESS LEVELS}

Using the protocols and tools previously described, the best LIGO surface cleanliness levels achieved to date are of the order of $\sim 100$, refer to Table 4 . This cleanliness level was obtained from a 4" silicon wafer (witness plate) which was placed in a vertical orientation adjacent to one of the core optics just before pump down and removed shortly after the next vent. The sample was subsequently scanned in-house at 10X magnification on a Nikon LV100 Microscope. The single image obtained was then analyzed using NIS-Elements AR imaging software with exposure set at $114 \mathrm{~ms}$. At the time of writing the authors were working on an updated analysis method for these samples, which will be reported in a future paper.

Table 4 summarizes the cleanliness levels collected from one area within the LIGO UHV over 15 months, and indicates the progress made during this period that included four separate periods under vacuum. Each of these periods under vacuum is referred to as 1 cycle. If work was performed in chamber after the wafer was placed or before the wafer was retrieved it is also indicated e.g. 1 cycle plus minimal work indicates that the wafer was exposed in chamber for one pump down and subsequent vent as well as some work either ahead of close out or after venting. During these vent periods the installation teams applied the mitigation steps summarized in this paper. The detail associated with these cleaning session can be summarized as follows: -

(i) Aug 2012 (just before pump down \#1) - Basic wipe down of exposed surfaces. Estimated $\sim 1$ hour.

(ii) Dec 2012 (just before pump down \#2) - Multiple wipe downs over a 2 week period up to 30 minutes each time.

(iii) Jun 2013 (just before pump down \#3) - In depth wipe down of all horizontal \& vertical metal surfaces including hard to reach areas $\sim 1-1.5$ hours per open chamber. 
Table 4. Cleanliness levels collected from one area within the LIGO UHV over 15 months.

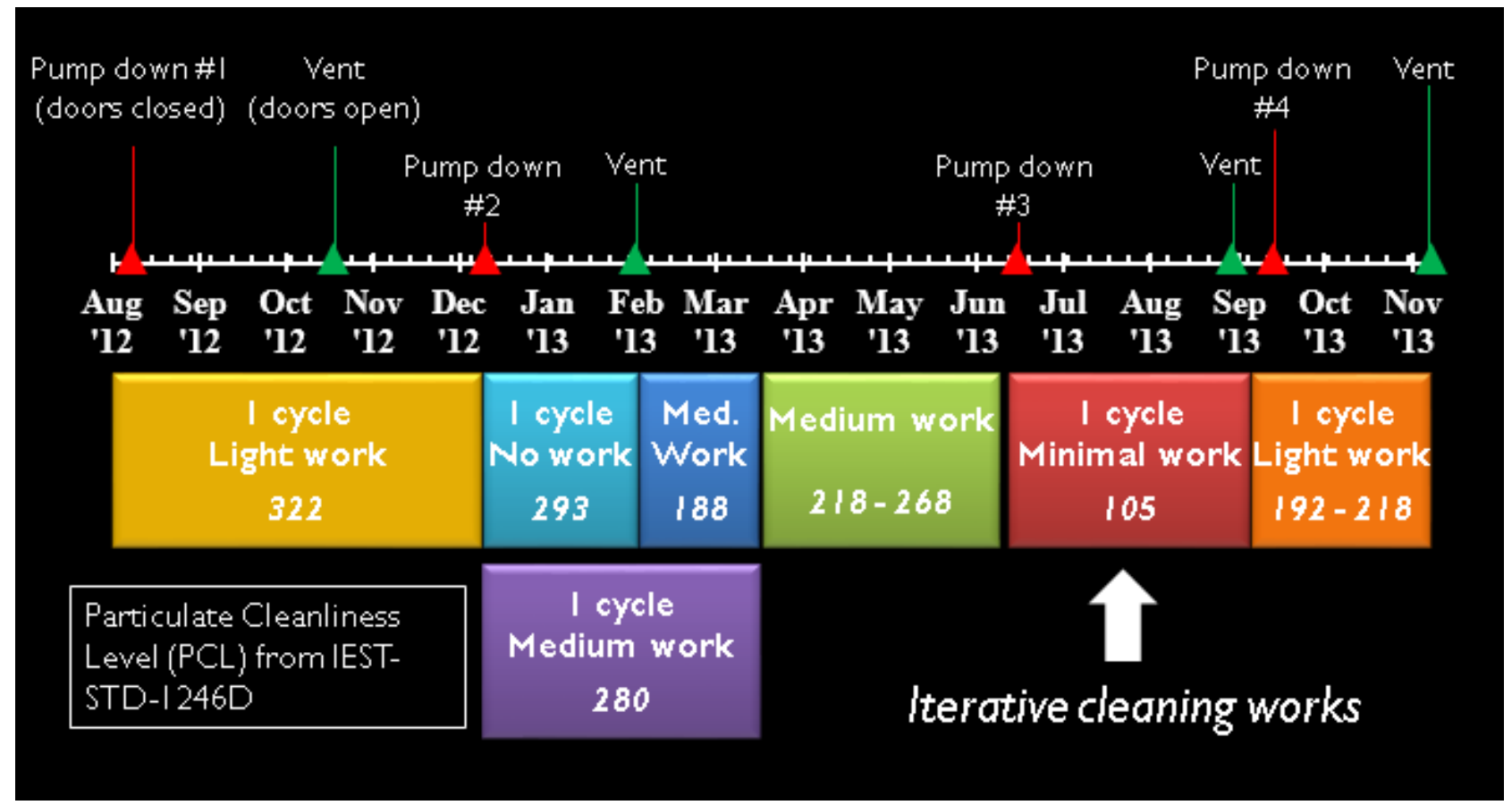

The message this gives is that iterative cleaning works and while further work is required to match LIGO's requirement, current results are within an achievable factor of 2 of requirements. LIGO's progress thus far is a quantifiable factor of 3 in cleanliness level class, as per IEST-STD-1246-D [16], which corresponds to about 2 orders of magnitude reduction in particle density [44].

\section{FUTURE WORK}

\subsection{Air Knife / Nozzle \& Optic Illumination System}

The vacuum chamber pump-down (transition from atmosphere to vacuum) as well as the vent (vacuum to atmosphere) cycles cause the migration and redistribution of particles within the vacuum chambers [45]. In addition, there is a finite, and not insignificant amount of time between the removal of particulates from critical optical surfaces with FC and pump-down. As a consequence, despite best cleaning efforts, there is a finite risk that a significant areal density of particulates may be found within the central region of the laser beam. These particulates can lead to pin-hole damage to the optic coating. LIGO can only tolerate a limited number of such events/cycles before the integrated loss due to coating damage becomes intolerable. Ideally LIGO would like to remove the particulates which migrate onto the critical optical surfaces after the pump-down. The NIF project [46] has implemented air knife systems to blow particulates off of some of their optical surfaces in air. LIGO is pursuing the development of in-vacuum air knife/nozzle systems for the critical optical surfaces. The concept is to create a hypersonic jet(s) of ionized nitrogen (or air) directed at the critical optical surface. A vacuum system with a small (3" dia.) suspended optic (single stage) has been set up to test and optimize the parameters of such a system (flow rate, pressures, stand-off distance, ion density, etc.). Assembly of the vacuum system has just recently been completed and testing with the system will start soon. [47]. 


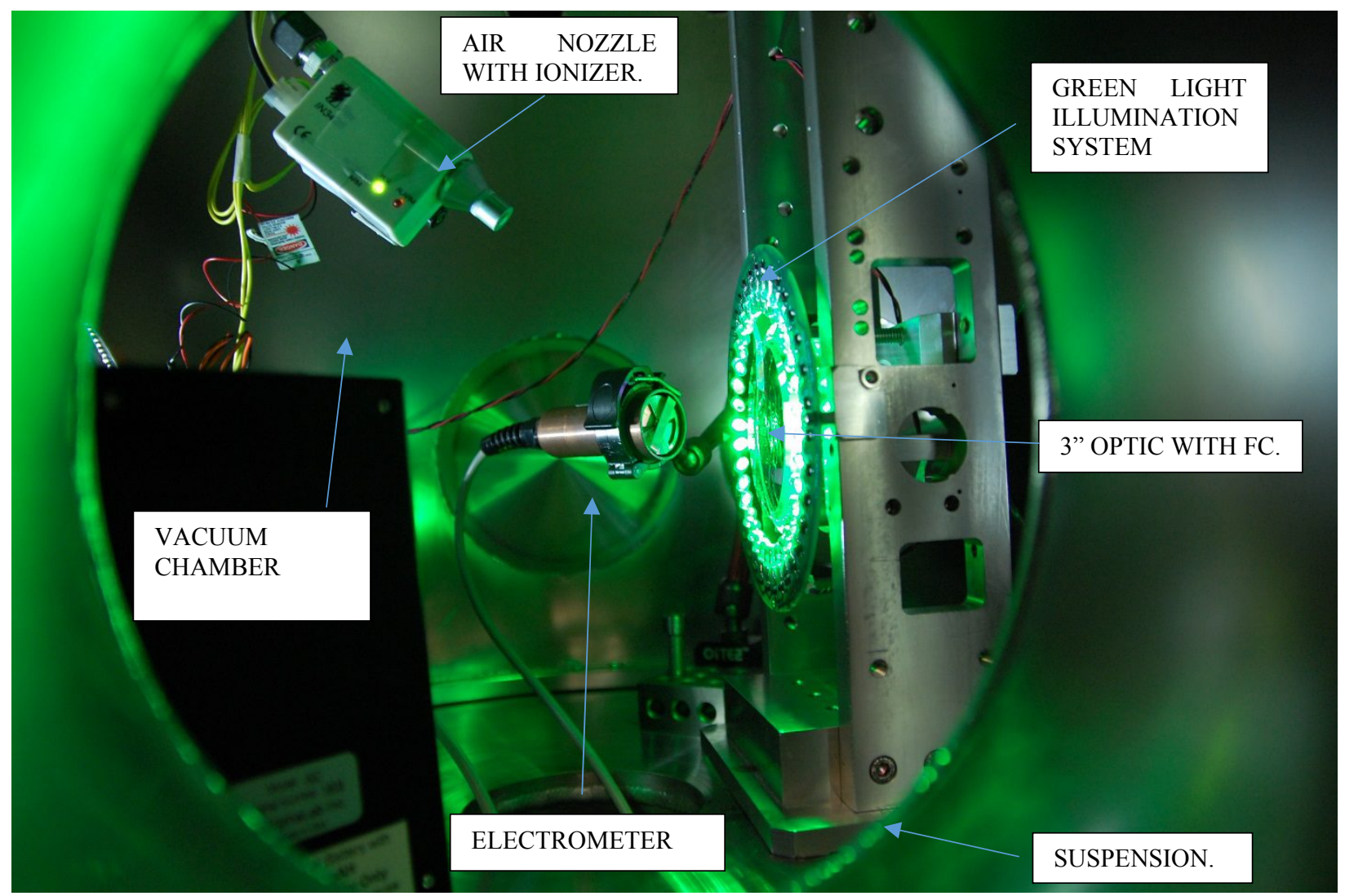

Figure 17. Air Nozzle Vacuum \& Optic Illumination System - Test System.

\subsection{Laser Induced Damage Threshold (LIDT) Assessment / Research}

Ion Beam Sputtering (IBS) coated, super polished, 1" fused silica optics are cleaned with First Contact, stored in clean PET-G containers, and sent to the observatories for placement in the UHV chambers. These witness samples collect contamination, typically from just before pump-down to just after the subsequent vent. After removal from chamber, they are placed in their clean containers, documented, and shipped to Caltech for analysis [48]. At Caltech, an initial analysis of the sample is carried out with a Nikon LV100 Microscope using NIS-Elements AR Imaging software, and the collected data is stored on the DCC for comparison with subsequent scans. In order to irradiate the 1" optic samples (with site dust) a $1064 \mathrm{~nm} \mathrm{CW}$ laser with a $50 \mu \mathrm{m}$ waist has been procured and setup. A mechanical stage raster scans the beam over a $1 \mathrm{~cm}$ square area with $100 \%$ coverage. This is currently performed in air under a laminar flow bench. Between each irradiation the optic is scanned in the Nikon NIS-Elements system.

Now that installation is complete at both sites vacuum incursions will now be at a minimum. As a result, a system to make artificial dust samples for testing has been developed. (Even during the LIGO installation period the time between subsequent vents could be 6 to 9 months.) From the knowledge we have on the make-up of the dust at both sites a dust soup is prepared in a stainless steel container, these containers are then stored in a desiccant cabinet. When ready a small amount of this dust is placed into a modified funnel, which has a 5 micron stainless steel mesh held between viton orings. Using a knife to tap on the funnel the dust is then shaken onto the 1" optic sample. During this last step the optic sample is illuminated with one of our flashlight arrays.

As an example of the sort of imagery (and data that can be extracted, collected and compared) consider a witness sample that was created artificially by applying $5 \mu \mathrm{m}$ aluminum dust onto $1 "$ optic with a stainless steel shaker. After an initial scan at Caltech on the Nikon NIS-Elements system an area of $5 \mathrm{~mm} \times 5 \mathrm{~mm}$ was irradiated at $\sim 450 \mathrm{~W} / \mathrm{mm} 2$ and then rescanned on the microscope. Figure 18 below shows two of these particles in both its pre and post irradiation form [49]. 


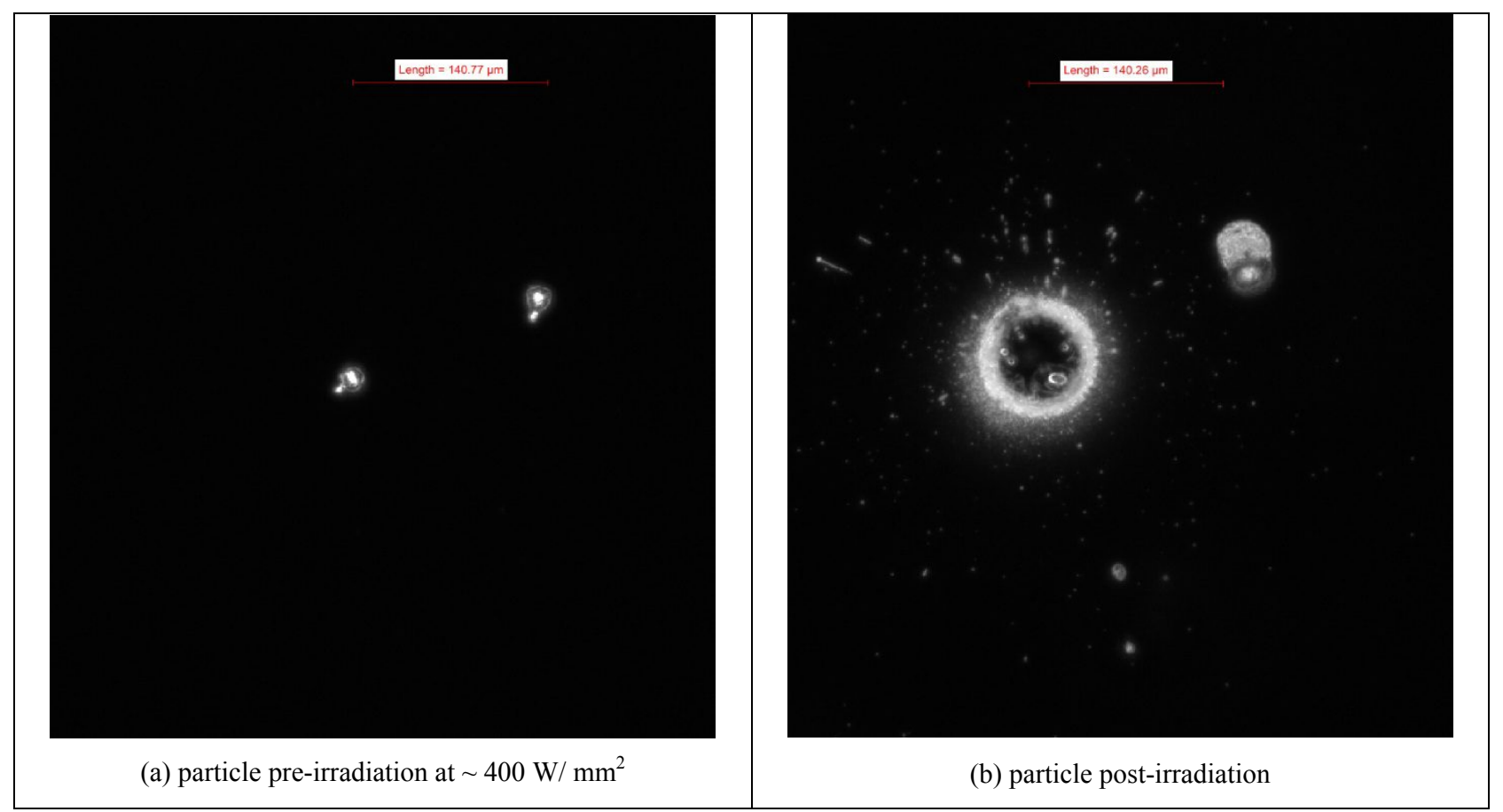

Figure 18. Example of Particulate-Initiated, Laser-Induced Coating Damage.

Initial results [50] from testing to date indicates that the onset of particulate induced melting of the coating occurs at $\sim 92$ $\mathrm{W} / \mathrm{mm}^{2}$. For comparison the peak fluence at high power will be $96 \mathrm{~W} / \mathrm{mm}^{2}$ for the larger optics and up to $2000 \mathrm{~W} / \mathrm{mm}^{2}$ on some of the smaller optics. As can be seen in the example above not only is pin-hole damage in the optic coating induced but also any cleanliness levels previously achieved will be adversely affected. The assessment of LIDT within LIGO as a result of particulate contamination has only just begun. Now that the experiment and the analysis system is setup over the next year or so LIGO staff will work on the important (key) milestone in this work which is the matching up of the damage levels to the cleanliness (PCL) of the optic (or obscuration ratio of the optic).

\section{CONCLUSIONS}

The cleaner LIGO is, the better the ultimate performance. Mitigation \& Remedial Clean Up are the keys. As indicated in this paper iterative cleaning improves the end result. As a result of these ongoing efforts, LIGO now has a better understanding of the contamination levels (cleanliness levels achieved to date are of the order of $\sim 100$ ) and has made significant improvements to methods of controlling contamination and protecting the optics from damage in the Advanced LIGO Interferometers. If LIGO fail to achieve the contamination goals, it is not a fail (or a 'fall from a cliff'), there will instead be a degradation to performance especially at high power (in $\sim 2$ years' time). Integrated testing can continue with the current level of particulates. Ultimately it will be a trade-off between limitations of increasing loss and downtime due to more frequent interventions. As mentioned above LIGO will work over the next year or so on the important (key) milestone of matching up the damage levels to the surface cleanliness levels of the optic (or obscuration ratio of the optic) and to develop the in-vacuum air knife/nozzle systems for the critical optical surfaces.

\section{ACKNOWLEDGEMENTS}

The authors gratefully acknowledge the support of the United States National Science Foundation (award No. PHY-07 57058). LIGO was constructed by the California Institute of Technology and Massachusetts Institute of Technology with funding from the National Science Foundation, and operates under cooperative agreement PHY-0107417. Caltech also acknowledges financial support from the Science and Technology Facilities Council of the United Kingdom. This paper filed internally under LIGO document number LIGO- P1400205. LIGO Internal Reports may be accessed at https://dcc.ligo.org/cgi-bin/DocDB/DocumentDatabase. The authors wish to acknowledge the many colleagues in LIGO and the LIGO Scientific Collaboration who have supported this work. In particular, the authors would like to thank Jeff Bartlett, Garilynn Billingsley, Dennis Coyne, Eric Gustafson, Matthew Heintze, Norna Robertson, Betsy Weaver and 
Liyuan Zhang within LIGO. In addition, the authors would like to thank all of the excellent contacts they now have within the National Ignition Facility.

\section{REFERENCES}

All of the LIGO documents listed below are public (unless stated as "LIGO internal report") and as mentioned above are available from at the LIGO public database here: - https://dcc.ligo.org/cgi-bin/DocDB/DocumentDatabase. For LIGO internal reports contact authors of this paper.

[1] Einstein, A., "Die Grundlage der Algemeinen Relarivitatstheorie," Annalen der Physik, 49, 769, (1916).

[2] Harry, G. M., "Advanced LIGO: The Next Generation of GW Detectors," Class. Quantum Grav. 27(8), 084006, (2010).

[3] Hulse, R., A., J.H. Taylor, Astrophys. J., 195, (1975), L51

[4] Taylor, J., H., Rev. of Mod. Phys., 66, (1994), 711.

[5] Rubbo, L., J., Larson, S., L., Larson, M., B., Zaleski, K., D., "Gravitational Waves: new observatories for new astronomy," arXiv:physics/0509201, (2005).

[6] Fritschel, P., on behalf of the LIGO Scientific Collaboration, to be submitted to a special issue of Classical and Quantum Gravity. (LIGO DCC Number P1400177, Oct 17, 2014.)

[7] Abbott, B. et al., "LIGO: the Laser Interferometer Gravitational-Wave Observatory," Rep. Prog. Phys., 72, 076901, (2009).

[8] The LIGO Scientific Collaboration \& the Virgo Collaboration, (2009), [Nature], 460990.

[9] Abadie, J. et al., (The LIGO Scientific Collaboration and the Virgo Collaboration), "Search for Gravitational Waves from Low Mass Compact Binary Coalescence in LIGO's Sixth Science Run and Virgo's Science Runs 2 and 3," Phys. Rev D, 85, (2012), 082002.

[10] Abadie, J. et al., (The LIGO Scientific Collaboration and the Virgo Collaboration), "All-sky search for gravitationalwave bursts in the second joint LIGO-Virgo run," Phys. Rev. D, 85, (2012), 122007.

[11] Aasi, J. et al., (The LIGO Scientific Collaboration and the Virgo Collaboration), "Gravitational-waves from known pulsars: results from the initial detector era," Astrophys. J., 785, (2014), 119.

[12] Gushwa, K., Bartlett, J., Torrie, C., Hanford Contamination Control Update - Back to Basics, LIGO-G1400378-v2, Oct 21, 2014.

[13]Zucker, M., Protecting installed core optics from particulates, LIGO-T080067-v1, Nov 1, 2013

[14] Coyne, D., Particulate Contamination Control Requirements, LIGO-T1300511-v11, Jun 5, 2013.

[15] Billingsley, G. et al, Core Optics Components (COC) Design Requirements Document (DRD), LIGO-T000127-v2, Jul 23, 2010.

[16] Stowers, IF IEST-STD-1246D, "Product Cleanliness Levels and Contamination Control Program," Institute of Environmental Sciences and Technology, 940 E. Northwest Highway, MT. Prospect, IL 60056.

[17] Stowers, IF., and Ravizza, DL., "The Particle Cleanliness Validation System", Proceedings 48th Annual Technical Meeting of the Institute of Environmental Science and Technology, April 28-May 1, 2002, UCRL-JC-145932.

[18] Private Communication with LIGO.

[19] MSL Contamination Control Requirements. Private Communication with LIGO.

[20] International Standard ISO 14644-1, "Cleanroom and associated controlled environments - Part 1: Classification of air cleanliness," May 1, 1999.

[21] LIGO Internal Report - LIGO reference E1300147.

[22] LIGO Internal Report - LIGO reference L1300087.

[23] Coyne, D., LIGO Vacuum Compatible Materials List, LIGO-E960050-v13, Jun 19, 2014.

[24] Coyne, D., Torrie, C., Metal components intended for use in the Adv LIGO Vacuum System, LIGO-E0900364-v8, Mar 25, 2011.

[25] Coyne, D., Qualifying Parts for LIGO UHV Service, LIGO-E1000088-v1, Apr 20, 2010.

[26] Bland, B., Coyne, D., Fauver, J., LIGO Clean and Bake Methods and Procedures, LIGO-E960022-v25, Jul 3, 2012.

[27] ASTM B479-06, "Standard Specification for Annealed Aluminum and Aluminum-Alloy Foil for Flexible Barrier, Food Contact, and Other Applications", [ASTM International], West Conshohocken, PA, 2006, www.astm.org (sections 3.1.4 and 10.3.1).

[28] Coyne, D., Desiccants acceptable for use with LIGO Parts, LIGO-E1300075-v2, Sept 4, 2014, LIGO Internal Report. 
[29] Military Specification, "Desiccants, Activated, Bagged, Packaging Use and Static Dehumidification," MIL-D3464E.

[30] Coyne, D. Long Term Storage Plan for the Components of the Third Advanced LIGO Interferometer, LIGOE1300001-v11, Jun 18, 2014, LIGO Internal Report.

[31] Jones, L., Torrie, C., Gerfen, M., Galling Tendencies and Particles Produced by Ultra Clean Screw Threads, LIGOT040111-v1, Jul 09, 2013.

[32] Coyne, D., Generic Requirements \& Standards for Detector Subsystems, LIGO-E010613-v2, Jan 2, 2013.

[33] Gushwa, K., Fauver, F., Phelps, M., Torrie, C., Prudential Body Box Results, LIGOT1300093-v7, Nov 01, 2013.

[34] Bland, B., Fauver, J., Heintze, M., Torrie, C., Coyne, D., aLIGO Contamination Control Plan, LIGO-E0900047-v21, Feb 20, 2013.

[35] Phelps, M., Gushwa, K., Torrie, C., Optical contamination control in the Advanced LIGO ultra-high vacuum system, LIGO-P1300192-v2, Mar 11, 2014.

[36] Sanchez, E., ALIGO, INS, Flashlight Holder Assembly (Flashlight Array), LIGO-D1300223-v3, Sep 3, 2014.

[37] LIGO Internal Report - LIGO reference T1400024.

[38] Internal Memo in LIGO project.

[39] LIGO Internal Report - LIGO reference M1300383.

[40] Torrie, C., LIGO Contamination Control Training Material (LIGO Internal Document), LIGO-E1400029-x0, Jun 27, 2014.

[41] Billingsley, G., Gushwa, K., Torrie, C., Collection of Documents associated with cleaning optics for advanced, LIGO-E1400363-x0, Sep 18, 2014.

[42]Phelps, M., Gushwa, K., Torrie, C., Billingsley, G., Abbott, B., Sanchez, E., Protective devices and methods for precision application of cleaning polymer to optics, US Patent Publication number US20140196748 A1, 17 July, 2014.

[43] Sanchez, E., ALIGO, COC, ETM/ITM FC Spray Guard Assembly Drawing, LIGO- D1200490-v5, Sep 16, 2014.

[44] Gushwa, K., Torrie, C., Summary of 4” Wafer Data from LIGO, LIGO-G1400142-v1, Mar 11, 2014.

[45] LIGO Internal Report - LIGO reference G0900913, T1300393 and T1300394.

[46] Gourdin, W. H., Dzenitis, E., Martin, D., Listiyo, K., Sherman, G., Kent, W., Butlin, R., Stolz, C. J., Pryatel, J., "Insitu surface debris inspection and removal system for upward-facing transport mirrors of the National Ignition Facility,” Boulder Damage Symposium XXXVI, Nov 11, 2004.

[47] Gushwa, K., Torrie, C., Billingsley, G., Abbott, B., Sanchez, E., Optic Green Light Illumination System (aka The Green Lantern), Provisional patent application was filed on Sep 12, 2014, Patent App CIT 6990-P.

[48] Torrie, C., Gushwa, K., Coyne, D., Contamination Sample Handling - How to receive, use, send, buy and store samples, LIGO-E1201096-v5, Jun 19, 2014.

[49] LIGO Internal Report - LIGO reference T1400462.

[50] Billingsley, G. et al, Laser Induced Damage due to Particulate Contamination, LIGO-G1400209-v2, Sep 8, 2014. 